\title{
DESENVOLVIMENTO E AVALIAÇÃO DE UM FITOCOSMÉTICO UTILIZANDO EXTRATOS E ÓLEO ESSENCIAL DE Aniba canelilla
}

\author{
L. I. SILVA ${ }^{1}$, G. F. SILVA ${ }^{1}$, P. M. ALBUQUERQUE ${ }^{1}$ \\ ${ }^{1}$ Universidade do Estado do Amazonas, Escola Superior de Tecnologia, Curso de Engenharia \\ Química \\ E-mail para contato: larissa.ipuchima@ gmail.com
}

\begin{abstract}
RESUMO - O uso de plantas da floresta Amazônica para a produção de fitocosméticos tem se tornado comum entre as grandes indústrias de higiene e limpeza. Este trabalho visou o desenvolvimento de uma emulsão cosmética com ativo natural de A. canelilla, espécie amazônica produtora de óleo essencial de interesse. Foram elaboradas diferentes formulações utilizando ativos de A. canelilla, verificando a estabilidade físico-química e as características organolépticas das emulsões. O potencial antioxidante das formulações foi avaliado pelo método do sequestro do radical DPPH. Foram preparadas quatro emulsões distintas contendo extratos hidroalcoólicos de galhos, de folhas, BHT (antioxidante padrão) e um controle; Os resultados obtidos demonstram que os extratos hidroalcoólicos apresentam compatibilidade química entre os componentes da formulação e podem ser incorporados em emulsões cosméticas como antioxidantes apresentando boa estabilidade.
\end{abstract}

\section{INTRODUÇÃO}

O desenvolvimento científico e tecnológico ao longo dos anos, vem oportunizando um grande avanço na pesquisa de produtos naturais. Impulsionado principalmente pelo apelo que a biodiversidade exerce sobre as pessoas, nota-se um grande aumento no uso de produtos naturais em substituição aos derivados minerais e animais (FUNARI; FERRO, 2005). A vegetação amazônica tem sido evidenciada neste contexto devido sua rica biodiversidade e grande potencial biotecnológico, dentre eles, para obtenção de fitocosméticos.

A adição de extratos, ceras, óleos vegetais e essenciais em produtos cosméticos agrega benefícios aos mesmos, pois apresentam diversas atividades biológicas devido à presença de metabólitos secundários como os fenóis simples, ácidos fenólicos, flavonoides, taninos, entre outros. Dentre as atividades clínicas atribuídas a esses componentes, pode-se citar ação antioxidante, antiinflamatória, antienvelhecimento e fotoprotetora que justificam o uso dos mesmos pela indústria cosmética. Seguindo esta tendência, surge a necessidade de estudos científicos que comprovem os efeitos benéficos desses componentes quando adicionados em diferentes formulações cosméticas, visando garantir a eficácia das mesmas (BALOGH, 2011). 


\section{9 a 22 de outubro de 2014 \\ Florianópolis/SC}

Neste estudo foi utilizada a Aniba canelilla, espécie amazônica de onde se extrai o óleo essencial de interesse, cujo principal componente é o 1-nitro-2-feniletano, responsável pelo odor semelhante ao da canela. Diversas atividades já foram descritas tais como, vasorelaxante, cardiovascular (INTERAMINENSE et al., 2013), antinociceptivo (LIMA et al., 2009), antioxidante (SILVA et al., 2007), antimicrobiana e citotóxica (SILVA, 2012).

Esta pesquisa visa o desenvolvimento de uma emulsão cosmética contendo extratos hidroalcoólicos obtidos de folhas e galhos de Aniba canelilla, que segundo Silva (2012) apresentou percentuais de inibição de mais de $90 \%$ dos radicais livres DPPH. Este estudo visa a comparação com formulações elaboradas com o antioxidante comumente utilizado em cosméticos butilhidroxitolueno, BHT, por meio da determinação das características macroscópicas, físico-químicas e estabilidade preliminar das emulsões, favorecendo o uso sustentável da biodiversidade amazônica utilizando-se do potencial amazônico para formulação de um produto fitocosmético.

\section{MATERIAIS E MÉTODOS}

As fases hidroalcoólicas dos extratos etanólicos de galhos e folhas de Aniba canelilla foram obtidos por maceração a frio no âmbito do projeto Bionorte (SILVA, 2012) e realizada a atividade antioxidante frente ao radical DPPH (BRAND-WILLIAMS et al., 1995; SANCHEZ-MORENO, 2002). O teste foi realizado adicionando-se $50 \mu \mathrm{L}$ de cada amostra teste em $1950 \mu \mathrm{L}$ de solução de DPPH· 0,06 mol/L seguido de homogeneização da mistura. Após 30 minutos de reação, a absorbância foi monitorada a um comprimento de onda de $517 \mathrm{~nm}$ sendo, os resultados expressos como porcentagem de inibição de DPPH. (SILVA et al., 2007) aplicando-se o teste ANOVA One Way para análise de variância seguida de Tukey para discriminação das diferenças.

As emulsões foram elaboradas pela técnica da inversão de fases em oito lotes diferentes sendo quatro em duplicata contendo Crodabase CR2 (35,0g), Isopropyl Myristate (17,5g), Dimethicone $(17,5 \mathrm{~g})$, Propylparaben $(0,50 \mathrm{~g})$, Mineral Oil (17,5g), Aqua (370,0g), Propylene Glycol $(10,0 \mathrm{~g})$, Disodium EDTA (0,50g), Methylparaben (0,75g), Glycerin $(20,0 \mathrm{~g})$, Imidazolidinyl Urea $(0,50 \mathrm{~g}) \mathrm{e}$ Essencial Oil (1,0ml). As quatro formulações foram divididas pelo antioxidante utilizado sendo: uma contendo BHT $(0,1750 \mathrm{~g})$, outra com extrato hidroalcoólico de galhos $(0,1750 \mathrm{~g})$, uma terceira com extrato hidroalcoólico de folhas $(0,1750 \mathrm{~g})$ e a última somente com glicerina como controle. As fases aquosas e oleosas foram aquecidas a $75^{\circ} \mathrm{C}$ vertendo-se posteriormente a fase aquosa à fase oleosa sob agitação constante em 1200rpm até o arrefecimento natural a temperatura ambiente onde os conservantes e antioxidantes foram adicionados. Após $24 \mathrm{~h}$ do preparo das emulsões, foram determinadas as características macroscópicas, físico-químicas e atividades antioxidante.

A análise macroscópica foi realizada após $24 \mathrm{~h}$ do preparo das formulações e durante as análises físico-químicas sendo observada a homogeneidade das formulações com a finalidade de identificar possíveis indícios de instabilidade tais como cremeação, floculação e coalescência (FERRARI, 1998). O teste de centrifugação foi realizado submetendo-se 10,0 g emulsões a ciclos de $1.000,2.500 \mathrm{e}$ $3.500 \mathrm{rpm}$ por 15min em cada rotação à temperatura ambiente (FERRARI, 1998; RIEGER, 1996).

A determinação da densidade específica foi feita com picnômetro, sendo avaliada a relação 


\section{9 a 22 de outubro de 2014 \\ Florianópolis/SC}

entre a massa da amostra e a massa da água, ambas a $20^{\circ} \mathrm{C}$ (FARMACOPÉIA BRASILEIRA, 2001).

$\mathrm{O}$ pH e a condutividade foram determinadas inserindo o eletrodo diretamente na solução aquosa 1:10 (p/p) das amostras (DAVIS, 1977).

A medida da espalhabilidade foi realizada posicionando-se uma lâmina de vidro em outra contendo $25 \mathrm{mg}$ da amostra. O raio formado pelo espalhamento da base cremosa foi medido e repetiuse o procedimento, sempre a intervalos de 1 minuto, adicionando-se pesos aferidos de $2,1 \mathrm{~g}, 2,1 \mathrm{~g}$ e 4,6g (FERNANDEZ-MONTES, 2005).

As emulsões classificadas macroscopicamente como estáveis após $24 \mathrm{~h}$ de sua manipulação foram submetidas aos testes preliminares de estabilidade: estresse térmico e ciclo gela-degela (ANVISA, 2004; FERRARI, 1998; FERRARI 2002). O estresse térmico consistiu em acondicionar as emulsões em frascos plásticos de poliestireno submetendo-as ao aquecimento na faixa de temperatura de 40 a $80^{\circ} \mathrm{C}$. O aumento da temperatura ocorreu de $5 \pm 1^{\circ} \mathrm{C}$ em $5 \pm 1^{\circ} \mathrm{C}$, mantendo-se por $30 \mathrm{~m}$ em cada temperatura. As análises foram realizadas ao término a $80^{\circ} \mathrm{C}$ após o arrefecimento natural das amostras à temperatura ambiente (FERRARI, 1998). O ciclo gela-degela ocorreu submetendo-se as formulações a variações de temperatura por um período de 12 dias. $\mathrm{O} 1^{\mathrm{o}}$ dia correspondera as $24 \mathrm{~h}$ após o preparo das formulações. As amostras foram submetidas a $4 \pm 2^{\circ} \mathrm{C} / 24$ horas em geladeira e $45 \pm 2^{\circ} \mathrm{C} / 24$ horas em estufa, completando assim, um ciclo. As leituras foram realizadas antes do início do teste e no final do $6^{\circ}$ ciclo (12 dias). Foram utilizados os mesmos parâmetros de avaliação do teste de estresse térmico (FERRARI, 1998).

\section{RESULTADOS E DISCUSSÕES}

As emulsões cosméticas formuladas, apresentaram aparente compatibilidade química aos extratos de Aniba canelilla. As características obtidas foram consideradas desejáveis pelo formulador tais como, emulsões homogêneas e brilhantes, com aspecto fino e macio ao toque. Após o estresse térmico e ciclo gela-degela, que visa acelerar interações físico-químicas entre as matérias-primas num reduzido espaço de tempo (RIEGER, 1996), a homogeneidade foi perdida e o produto adquiriu um aspecto heterogêneo, opaco e fibroso, tidos como defeitos sérios (ISAAC et al., 2008).

As amostras submetidas a centrifugação apresentaram aspecto normal e sem alteração comparando-as a análises macroscópicas do tempo zero ( $24 \mathrm{~h}$ após a formulação). O procedimento de centrifugação é eficaz na determinação da instabilidade dos produtos emulsificados, já que a simulação de aumento da força de gravidade pode promover separação de componentes de diferentes densidades, como nas fases óleo e água. Velocidades de rotação moderadas entre 2000 e $5000 \mathrm{rpm}$, durante curto período, são muito úteis para verificar o comportamento das gotículas emulsionadas frente à força da gravidade (RIEGER, 1996).

As emulsões caracterizadas como macroscopicamente estáveis após $24 \mathrm{~h}$ da formulação, foram submetidas aos estudos preliminares de estabilidade, por um período de 15 dias (ANVISA, 2004). As 
amostras foram submetidas ao ciclo gela-degela e estresse térmico. Os resultados das análises de densidade, $\mathrm{pH}$ e condutividade encontram-se na Tabela 1.

Tabela 1 - Dados dos parâmetros físico-químicos analisados 24 horas após a elaboração e após o estresse térmico e ciclo gela-degela. Densidade $(\mathrm{g} / \mathrm{mL})$, Condutividade $(\mu \mathrm{S} / \mathrm{cm})$.

\begin{tabular}{c|ccc|ccc|ccc}
\hline \multirow{2}{*}{ Amostra } & \multicolumn{3}{c}{$24 \mathrm{~h}$} & \multicolumn{4}{c}{ Estresse Térmico } & \multicolumn{3}{c}{ Ciclo Gela-degela } \\
& Dens & $\mathrm{pH}$ & Cond. & Dens & $\mathrm{pH}$ & Cond. & Dens & $\mathrm{pH}$ & Cond. \\
\hline \multirow{2}{*}{ Controle } & 0,970 & 4,22 & 1,99 & 0,968 & 4,13 & 1,85 & 0,966 & 4,40 & 2,03 \\
& 0,976 & 3,86 & 1,88 & 0,970 & 3,88 & 1,86 & 0,957 & 4,30 & 2,24 \\
\hline \multirow{2}{*}{ EHG } & 0,977 & 4,17 & 2,19 & 0,957 & 3,94 & 2,12 & 0,976 & 4,33 & 2,27 \\
& 0,971 & 3,90 & 2,09 & 0,974 & 3,85 & 2,21 & 0,959 & 4,21 & 2,30 \\
\hline \multirow{2}{*}{ EHF } & 0,965 & 4,02 & 2,06 & 0,947 & 3,95 & 2,05 & 0,966 & 4,33 & 2,27 \\
& 0,960 & 3,88 & 1,55 & 0,954 & 3,92 & 1,67 & 0,956 & 4,42 & 1,53 \\
\hline \multirow{2}{*}{ BHT } & 0,966 & 4,00 & 1,81 & 0,972 & 3,81 & 1,76 & 0,957 & 4,29 & 2,17 \\
& 0,974 & 3,63 & 1,78 & 0,975 & 3,83 & 1,48 & 0,980 & 4,30 & 1,83
\end{tabular}

As densidades das formulações não apresentaram diferenças significativa entre os lotes avaliados. Os valores medidos durante os ensaios de estabilidade preliminar também não diferem entre si nos produtos elaborados, conforme observado na Figura 1a.

$\mathrm{O}$ pH de uma formulação deve garantir a estabilidade dos ingredientes da formulação, sua eficácia e segurança (ANVISA, 2004), bem como ser compatível com os fluidos biológicos de acordo com a via de administração pretendida. A maior estabilidade dos sistemas dá-se quando estes são mantidos dentro de uma pequena variação de $\mathrm{pH}$. Desta forma, a diminuição progressiva da estabilidade dá-se quando o pH se afasta de seu limite ótimo (GENNARO, 2000).
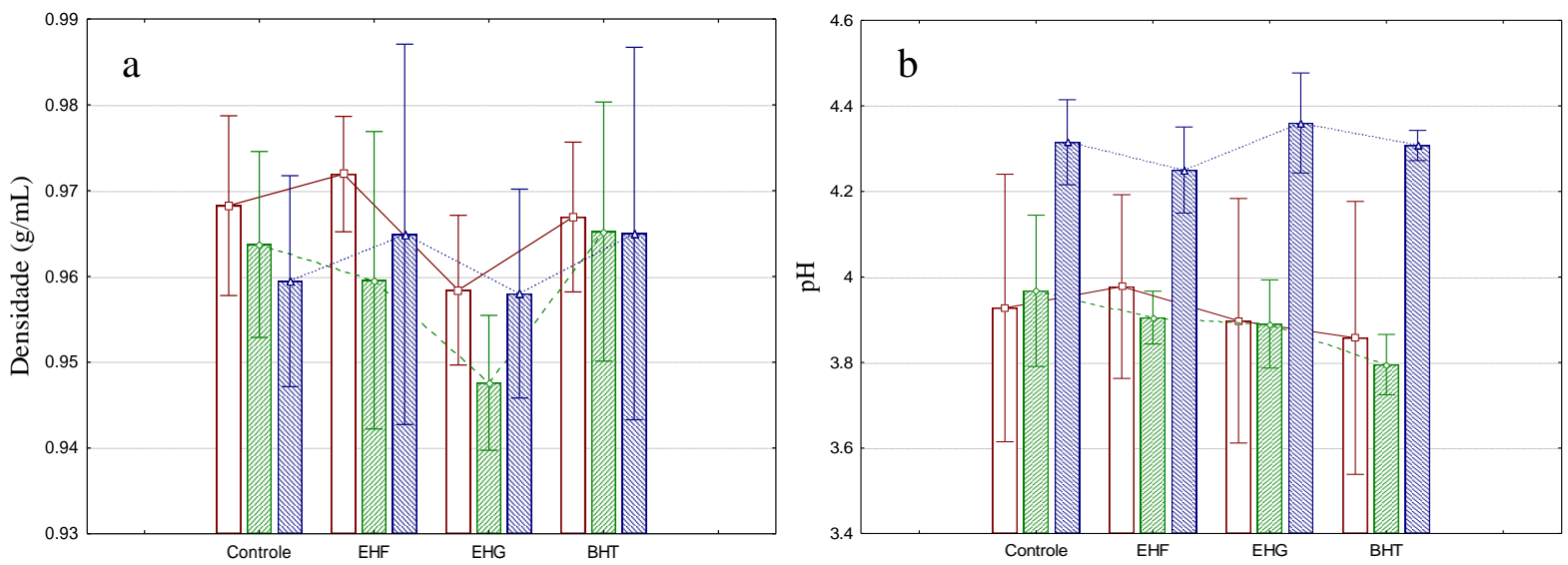

Controle: Sem antioxidante, EHF: Extrato Hidroalcoólico de Folhas, EHG: Extrato Hidroalcoólico de Galhos, BHT: Butilhidroxitolueno.

$\square 24 \mathrm{~h}$. Estresse Térmico $\mathbb{m}$ Ciclo gela-degela

Figura 1 - Gráfico das análises de: a - densidade $(\mathrm{g} / \mathrm{mL})$ sem diferença estatística entre os grupos e b análises de $\mathrm{pH}$ apresentando diferença estatística entre as formulações.

Apesar do valor do $\mathrm{pH}$ não ser uma medida tão sensível quando comparado à condutividade elétrica, alterações deste durante o período de estocagem nas diferentes 


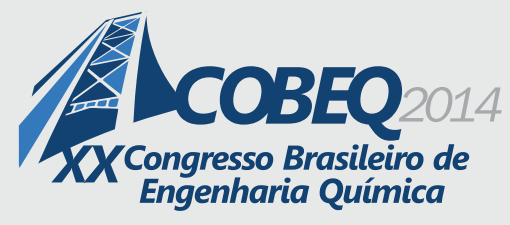

temperaturas podem indicar um processo de instabilidade. Segundo Masmoudi et al. (2005), a diminuição do $\mathrm{pH}$ pode representar uma oxidação da fase oleosa com formação de hidroperóxidos, ou mesmo a hidrólise de triglicerídeos levando à formação de ácidos graxos.

A condutividade elétrica apresentou valores constantes durante os ensaios de estabilidade preliminar, não havendo diferença estatística entre os valores observados conforme a Figura $2 \mathrm{a}$. A medição da condutividade elétrica é considerada como uma ferramenta útil para a avaliação da estabilidade da emulsão imediatamente após a sua preparação e depende também do grau de dispersão. As preparações O/A com partículas pequenas apresentam resistência reduzida: um aumento da resistência revela agregação de gotículas de óleo e consequentemente revela instabilidade. Uma emulsão fina de água num produto $\mathrm{A} / \mathrm{O}$ não conduz a corrente até que se verifique coalescência das gotículas, isto é, quando se torna instável (LACHMAN, 2001).

Avaliando a espalhabilidade das emulsões formuladas, notou-se que as manipulações Controle e BHT apresentaram valores maiores que as obtidas com os extratos vegetais, EHF e EHG, como mostra a Figura $2 b$.
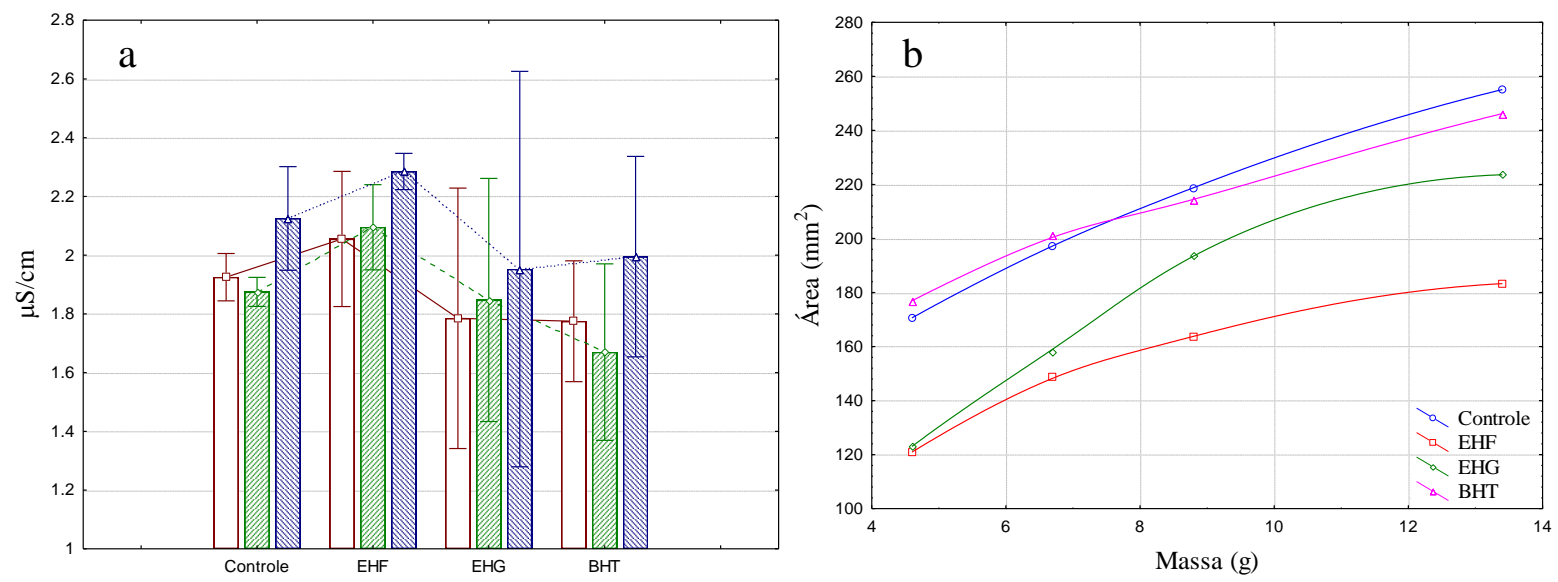

Controle: Sem antioxidante, EHF: Extrato Hidroalcoólico de Folhas, EHG: Extrato Hidroalcoólico de Galhos, BHT: Butilhidroxitolueno.

$\square 24 \mathrm{~h}$ Estresse Térmico Ciclo gela-degela

Figura 2 - Gráfico das análises de: a - condutividade elétrica $(\mu \mathrm{S} / \mathrm{cm})$ sem diferença estatística entre as formulações e os grupos e b - espalhabilidade apresentando diferença estatística entre os grupos.

Os dados referentes à espalhabilidade são importantes, pois se a emulsão produzida tiver melhores características reológicas, ela poderá melhorar a percepção sensorial no momento de sua aplicação sobre a pele e utilizar menor quantidade, levando a uma redução do consumo dessa preparação.

Estudos de Borella, et al. (2010) demonstraram que preparações obtidas sem aquecimento têm melhores características de espalhabilidade que aquelas obtidas com aumento da temperatura. Patil e Ravindra (2009) concluíram que o aquecimento utilizado na preparação da forma farmacêutica contendo óleo de alho afetava diretamente a atividade da droga. Sendo assim, 
a elaboração de emulsões cosméticas utilizando extratos hidroalcoólicos de $A$. canelilla podem ser estudadas preparando-as a frio, a fim de aumentar as características de espalhabilidade e ainda preservar os princípios ativos na preparação.

As atividades antioxidantes das formulações apresentaram-se iguais estatisticamente nas emulsões elaboras com os extratos da planta e o BHT e diferentes da emulsão elaborada sem o antioxidante como observado na Figura 3.

Os dados obtidos demonstram que os extratos hidroalcoólicos de A. canelilla mostraram-se eficientes como componentes antioxidantes na formulação, pois apresentaram valores iguais aos obtidos com o BHT, amplamente utilizado em cosméticos. Estes valores demonstram ainda que não houve incompatibilidade química entre os componentes da formulação mantendo a atividade antioxidante anteriormente observada por Silva (2012).

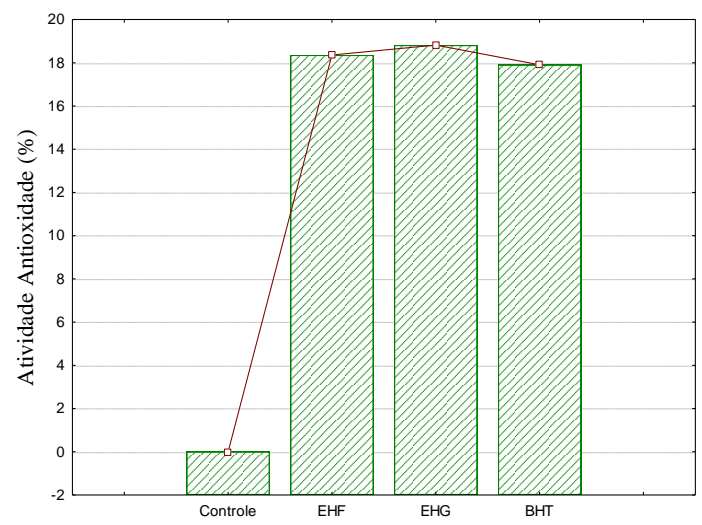

Figura 3 - Gráfico das análises de atividade antioxidante apresentando diferença estatística no grupo Controle: Sem antioxidante e BHT: Butilhidroxitolueno com EHF: Extrato Hidroalcoólico de Folhas e EHG: Extrato Hidroalcoólico de Galhos.

\section{CONCLUSÕES}

Os resultados obtidos mostram que emulsões cosméticas formuladas com extratos hidroalcoólicos de $A$. canelilla apresentam uma boa estabilidade físico-química comparando-as com as emulsões obtidas com BHT como antioxidante. A presença dos extratos não alterou os parâmetros físico-químicos das formulações, tornando-os uma ótima opção como ativo vegetal com potencial biotecnológico. A temperatura, por outro lado, deve ser controlada, pois altas temperaturas e variações bruscas da mesma podem comprometer a estabilidade dos produtos desestruturando a emulsão tornando-as heterogêneas e modificando o $\mathrm{pH}$ do meio podendo inativar ou até mesmo degradar componentes da formulação. As emulsões podem ser melhor preparadas a frio ou por meio da presença de substâncias que promovam uma aumento na espalhabilidade a fim de aumentar a veiculação de ativos vegetais e a percepção sensorial tornando-as mais finas e com menor pegajosidade, permitindo assim, obter emulsões com resultados mais satisfatórios. Um estudo aprofundado da estabilidade das emulsões deve ser conduzido, objetivando antever possíveis 
alterações das amostras ao armazená-las em condições que acelerem mudanças passíveis de ocorrer; permitindo, assim, obter informações sobre a estabilidade, segurança e eficácia do produto em menor tempo possível.

\section{REFERÊNCIAS}

ANVISA. Agência Nacional de Vigilância Sanitária. Guia de estabilidade de produtos Cosméticos. 1. ed. Brasília: Anvisa, v. 1, 2004.

BALOGH, T. S. Uso cosmético de extratos glicólicos: avaliação da atividade antioxidante, estudo da estabilidade e potencial fotoprotetor. 2011. Dissertação (Mestrado em Fármacos e Medicamentos). Universidade de São Paulo. São Paulo, 2011.

BORELlA, J. C.; RIBEIRO, N. S.; TEIXEIRA, J. C. L.; CARVALHO, D. M. A. Avaliação da espalhabilidade e do teor de flavonoides em forma farmacêutica semissólida contendo extratos de Calendula officinalis L . (Asteraceae). Rev. de Ciênc. Farm. Bás. e Aplic., v. 31, n. 2, p. 193-197, 2010.

BRAND-WILLIAMS, W.; CUVELIER, M. E.; BERSET, C. Use of a free radical method to evaluate antioxidant activity. LWT - Food Sci. and Technol., v. 28, n. 1, p. 25-30, jan. 1995.

DAVIS, H. M. Analysis of creams and lotions. In: Sezel AJ. Newburger's manual of cosmetic analysis.Washington: Association of Official Analytical Chemists; 1977.

FERNÁNDES-MONTES, E. A. Técnicas y procedimientos en formulación magistral dermatológica. Madrid: E. Alía, 2005.

FARMACOPÉIA BRASILEIRA. 4 ed. p. 2. São Paulo:Atheneu, 2001.

FERRARI, M. Desenvolvimento e avaliação da eficácia fotoprotetora de emulsões múltiplas contendo metoxicinamato de etilexila e óleo de andiroba (Carapa guianensis). 2002. 142p. Tese (Doutorado em Ciências Farmacêuticas), Universidade de São Paulo, Ribeirão Preto, 2002.

FERRARI, M. Obtenção e aplicação de emulsões múltiplas contendo óleo de andiroba e copaíba. Dissertação (Mestrado em Fármacos e Medicamentos). Universidade de São Paulo, Ribeirão Preto, 1998.

FUNARI, C. S.; FERRO, V. O. Uso ético da biodiversidade brasileira: necessidade e oportunidade. Rev. Bras. de Farmacog., v. 15, n. 2, p. 178-182, jun. 2005.

GENNARO, A. R. Remington: The science and practice of pharmacy. ed. 20, Philadelphia: Lippincott Williams e Wilkins, 2000. 


\section{9 a 22 de outubro de 2014 \\ Florianópolis/SC}

INTERAMINENSE, L. F. L.; RAMOS-ALVES, F. E. S.; XAVIER, R. J. B.; DUARTE, F. E.; MAGAlHÃES, G. P.; CALDAS, P. J.; MAIA, J. G. S.; SOUSA, P. J. C.; LAHLOU, S. Vasorelaxant effects of 1-nitro-2-phenylethane, the main constituent of the essential oil of Aniba canelilla, in superior mesenteric arteries from spontaneously hypertensive rats. Eur. J. Pharmac. Scienc., v. 48, n. 4-5, p. 709-16, 12 mar. 2013.

ISAAC, V. L. B.; CEFALI, L.C. C.; CHIARI, B.G.; OLIVEIRA, C.C.L.G.; SALGADO, H.R.N.; CORREA, M.A. Protocolo para ensaios físico-químicos de estabilidade de fitocosméticos. Rev. de Ciênc. Farmac. Bás. e Aplic., v. 29, n. 1, p. 81-96, 2008.

LACHAMAN, L.; LIEBERMAN, H. A.; KANIG, J. L. Teoria e Prática na Indústria Farmacêutica. v. 2, Fundação Calouste Gulbenkian, 2001.

LEONARDI, G. R.; GASPAR, L. R.; CAMPOS, P. M. B. G. M. Estudo da variação do pH da pele humana exposta à formulação cosmética acrescida ou não das vitaminas $\mathrm{A}$, $\mathrm{E}$ ou de ceramida, por metodologia não invasiva. Anais Bras. de Dermat., v. 77, n. 5, out. 2002.

LIMA, A. B.; SANTANA, M. B.; CARDOSO, A. S.; SILVA, J. K. R.; MAIA, J. G. S.; CARVALHO, J. C. T.; SOUSA, P. J. C. Antinociceptive activity of 1-nitro-2-phenylethane, the main component of Aniba canelilla essential oil Pergentino Jos e. Phytomedicine: Int. J. Phytot. and Phytopharmac. v. 16, n. 6-7, p. 555-559, jun. 2009.

MASMOUDI, H.; DRÉAU, Y. L. E.; PICCERELLE, P.; KISTER, J. The evaluation of cosmetic and pharmaceutical emulsions aging process using classical techniques and a new method: FTIR. Intern. J. of Pharm., v. 289, n. 1-2, p. 117-31, 31 jan. 2005.

PATIL, R.; RAVINDRA, R. Formulation development and evaluation from garlic oil macerate. The Inter. J. of Nut. and Wellness. v. 8, n. 1, 2009.

RIEGER, M. M. Teste de estabilidade para macroemulsões. Cosmet. Toiletries, v. 8, n. 5, p. 4753, 1996.

SANCHEZ-MORENO, C. Review: Methods Used to Evaluate the Free Radical Scavenging Activity in Foods and Biological Systems. Food Sci. and Tech. Internat., v. 8, n. 3, p. 121-137, 1 jun. 2002.

SILVA, G. F. Estudo do potencial biotecnológico de Aniba canelilla (H.B.K) Mez para obtenção de cosméticos. 2012. Dissertação (Mestrado em Biotecnologia e Recursos Naturais). Escola Superior de Ciências da Saúde. Universidade do Estado do Amazonas. Manaus, 2012.

SILVA, J. K. R.; SOUSA, P. J. C.; ANDRADE, E. H. A.; MAIA, J. G. S. Antioxidant capacity and cytotoxicity of essential oil and methanol extract of Aniba canelilla (H.B.K.) Mez. J. Agric. Food. Chem., v. 55, n. 23, p. 9422-6, 2007. 